\title{
The effect of cooling on the vibrotactile sensitivity of the tongue
}

\author{
BARRY G. GREEN \\ Monell Chemical Senses Center, Philadelphia, Pennsylvania
}

\begin{abstract}
Two experiments were carried out to investigate the effect of cooling on the sensitivity of the tongue to vibration. In the first experiment the temperature of the tongue was held at $36^{\circ}, 28^{\circ}$, or $20^{\circ} \mathrm{C}$ while the thresholds for detecting 30 - and $250-\mathrm{Hz}$ vibrations were measured using a twoalternative forced-choice paradigm. Cooling the tongue to $20^{\circ}$ reduced the sensitivity to $250-\mathrm{Hz}$ vibration but did not disturb the sensitivity to $30-\mathrm{Hz}$ vibration. The second experiment established that cooling continued to reduce the sensitivity to the $250-\mathrm{Hz}$ stimulus even when intensity levels were raised to $30 \mathrm{~dB}$ SL. The results are discussed in relation to (1) which types of mechanoreceptors mediate the perception of vibration on the dorsal surface of the tongue, (2) the effect of a rigid surround on lingual vibrotactile sensitivity, and (3) the possible impact of temperature on perception of the mechanical characteristics of foods.
\end{abstract}

Tactile information from the tongue is important for both the manipulation and the sensory assessment of ingested foods. In recognition of this fact and because of the presumed importance of tactile feedback from the tongue during speech production, a relatively large literature has developed on lingual mechanical sensitivity. However, many of the basic properties of tactile sensibility on the tongue remain to be explored. The present study examined one such property: the effect of skin temperature on the sensitivity to vibration. Because it was known that cooling the skin of either the hand or the arm could significantly reduce its sensitivity to high-frequency vibration (Bolanowski \& Verrillo, 1982; Green, 1977; Koradecka, 1974; Weitz, 1941) and, to a much lesser extent, its sensitivity to low-frequency vibration (Bolanowski \& Verrillo, 1982), it seemed likely that cooling might also reduce the sensitivity to vibration on the tongue.

Determining whether or not temperature affects lingual tactile sensitivity is relevant to a number of practical and theoretical issues related to oral sensibility. The loss of vibrotactile sensitivity due to cooling could, for example, produce changes in the perceived texture of foods, which might in turn alter their palatability. It is already known that temperature can affect the intensity of gustatory (e.g., Bartoshuk, Rennert, Rodin, \& Stevens, 1982; Calviño, 1986; Green \& Frankmann, in press; McBurney, Collings, \& Glanz, 1973) and common-chemical (Green, 1986; Sizer \& Harris, 1985) sensations; hence, knowledge of how temperature may change tactile sensitivity is important for an eventual understanding of the influence of temperature on the oral components of flavor.

The author thanks Barbara Gelhard for her assistance in the collection and analysis of the data reported in this study. This research was supported in part by Grant NS20577 from the National Institutes of Health. The author's address is Monell Chemical Senses Center, 3500 Market Street, Philadelphia, PA 19104.
In addition, establishing whether there is a frequencyspecific effect of cooling will help clarify the confusing literature regarding which population of mechanoreceptors mediates detection of vibrotactile stimulation on the dorsal surface of the tongue. Verrillo $(1966,1968)$ found that when a vibrotactile stimulus was presented through a rigid surround (with a 1-mm gap), sensitivity on the dorsal surface was independent of the frequency of vibration. The flat frequency function Verrillo obtained with a surround was in sharp contrast to the U-shaped function that was typically obtained on the dorsum of the tongue when no surround was present (Sherrick, 1953; Verrillo, 1966). Verrillo reasoned that the surround prevented vibration delievered to the dorsal surface from reaching Pacinian corpuscles (PCs), which anatomical evidence had indicated lay in the dermis beneath the ventral surface of the tongue. It followed, then, that the flat threshold function was the result of stimulating a population of mechanoreceptors located in the dorsal surface of the tongue whose response was not frequency-dependent.

In the two decades since Verrillo's (1966) first study, a number of investigations of vibrotactile sensitivity on the tongue have appeared. Agreement among the studies as to the effect of vibration frequency has often been lacking. Supporting Verrillo's finding of a flat threshold function was an experiment reported by Fucci (1972) that compared the sensitivities of the tongue, palm, and lip when a rigid surround was used. The sensitivity of both oral structures remained constant between 60 and $900 \mathrm{~Hz}$. However, a number of studies seem to contradict Fucci's data, indicating instead that frequency was a factor even when a surround was used (e.g., Fucci, Blackmon, Lindsey, \& McCaffrey, 1974; Fucci, Crary, \& Telage, 1977; Fucci, Curtis, \& Harnack, 1974; Fucci, Hall, \& Weiner, 1971; Telage, Fucci, \& Annst, 1972; Telage, Fucci, \& Blackmon, 1976). Subsequent to those studies, Telage and Petrosino (1978) again obtained a relatively 
flat threshold function, which they attributed to the elimination of a tongue-clamping procedure that had been used in the previous studies by Fucci and his co-workers. This observation failed to explain why Fucci (1972) had originally obtained a flat threshold function while using the clamping procedure, or why Fucci and Petrosino (1982) found a very large (15-20 dB) effect of frequency on perceived intensity without using the clamping procedure. Clearly, the conditions under which frequency affects the sensitivity of the tongue to vibration have yet to be established. The present study was intended in part to provide another test of Verrillo's original finding by obtaining, with a rigid surround, thresholds for low- and high-frequency vibration while assessing the effect of cooling. Because PCs are known to be temperaturesensitive (Inman \& Peruzzi, 1961; Ishiko \& Loewenstein, 1961), if a reduction in sensitivity produced by cooling were to be limited to high frequencies it would imply that PCs can be activated by vibrations generated on the dorsal surface of the tongue, even when a surround is used.

The results of a study published a decade ago suggest that temperature has at least a minor influence on the sensitivity of the tongue to high-frequency vibration. Fucci, Crary, Wilson, and Curtis (1976) measured the threshold for detecting a $250-\mathrm{Hz}$ stimulus after subjects had repeatedly sipped aliquots of water that ranged in temperature from $17^{\circ}$ to $57^{\circ} \mathrm{C}$. Thresholds were reported to be an average of $2.4 \mathrm{~dB}$ higher following rinses with $17^{\circ}$ water than they were following rinses with $37^{\circ}$ water. This result is difficult to interpret, however, because Fucci et al. did not measure the temperature of the tongue, and the psychophysical procedure they employed required the tongue to be clamped outside the mouth for an unknown duration during testing. Exposing the tongue to ambient air without further temperature rinses undoubtedly reduced the impact of the rinses; the warmed tongue would tend to cool by evaporation and the cooled tongue would tend to warm due to normal blood flow. It may have been because of this procedure that cooling the tongue with $17^{\circ}$ rinses produced an effect that was much smaller ( $2.4 \mathrm{~dB}$ vs. $17.5 \mathrm{~dB})$ than an effect that had been obtained in a study on the hand in which the skin had seemingly been cooled less severely (to $20^{\circ} \mathrm{C}$; Green, 1977). Fucci et al. also investigated only one frequency of vibration, leaving unanswered the question of whether the weak thermal effect they found would extend to lowfrequency vibration.

The two experiments reported here, in which the temperature of the tongue was controlled and monitored on every trial, demonstrated that cooling the tongue to $20^{\circ} \mathrm{C}$ caused a large reduction in both threshold and supra threshold vibrotactile sensitivity on the anterior dorsal surface of the tongue. The experiments further showed that the effect of cooling was limited to high-frequency vibration.

\section{EXPERIMENT 1}

The purpose of this experiment was to measure vibrotactile thresholds on the anterior dorsal surface of the tongue when the tongue was either cooled or held at normal oral temperature.

\section{Method}

Subjects. Five adults $(2$ males and 3 females, average age $=$ 26.6 years) were paid to participate. All had served before in psychophysical experiments, but never in a vibrotactile detection task.

Apparatus and Procedure. The vibrotactile stimulus was produced by a B \& K 4810 minishaker driven by a Wavetek Model 193 function generator and a B \& K Type 2706 power amplifier. The driving voltage, and hence the amplitude of vibration, was controlled manually with step attenuators. Vibration amplitude was monitored with a BBN 501 accelerometer (which had been calibrated visually) that was encased in the base of the contactor. The contactor itself was constructed from a polyethylene tubing connector that had a diameter at one end appropriate for stimulating a localized area of the tongue $(0.6 \mathrm{~cm})$ and a diameter at the other end that enabled it to fit snugly over the barrel of the accelerometer. In order to produce a smooth stimulating surface that also had a low thermal conductivity, the hollow interior of the connector was packed with silicone glue. Before the glue hardened, a 40-ga copper-constantan thermocouple was threaded through a hole in the side of the connector and pushed up to what would become the stimulating surface of the contactor. The bare end of the thermocouple was then bent at a $90^{\circ}$ angle to make it lie flush with the surface, partially embedded in silicone and partially exposed to air. (This was accomplished by placing the connector upside down on a piece of lightly oiled glass so that the silicone, with the thermocouple in it, formed a smooth surface as it hardened against the glass.) Hence the finished contactor had a circular surface area of $0.28 \mathrm{~cm}^{2}$ with a thermocouple built in to enable measurement of skin temperature at the site of tactile stimulation. The output from the thermocouple was displayed on a digital thermometer, and the analog output from the thermometer was led to an A/D converter that was connected in turn to a Commodore 64 microcomputer. Skin temperature was therefore monitored both visually and by computer.

The vibrator was supported by a modified microscope stand that enabled adjustment of the height and angle of the contactor for each subject. A rigid surround, constructed from clear Plexiglas and containing a circular hole $0.8 \mathrm{~cm}$ in diameter, was supported by a separate adjustable stand. The vibrator was situated so that the contactor protruded through the surround by $1 \mathrm{~mm}$. Between trials a small water-circulated thermode rested against the surface of the contactor to maintain its temperature within a few tenths of a degree of the temperature at which the skin was being tested. (It was discovered in preliminary tests that despite the relatively low thermal conductivity of the contactor, the thermal load it produced at room temperature was sufficient to affect skin temperature during mechanical stimulation.) The thermode was lifted from the contactor just before each trial.

The subject sat facing the apparatus with a sink on his/her right. Also to the right were an ice bath and two circulated constanttemperature baths, each of which contained bottles of deionized water to be used as temperature rinses. Before each threshold measurement the subject rinsed repeatedly with cold water (about $\left.3^{\circ}-5^{\circ} \mathrm{C}\right)$, cool water $\left(20^{\circ} \mathrm{C}\right)$, or warm water $\left(38^{\circ} \mathrm{C}\right)$, holding the liquid toward the front of the mouth. The duration of a rinse was self-timed, with the subject told to count to 10 at a rate that estab- 
lished rinse duration at approximately $10 \mathrm{sec}$. The subject then expectorated, counted to 5 , and sipped again. This was repeated for 3 to $5 \mathrm{~min}$, depending upon the subject. (The time required to cool or warm the mouth to the target temperature varied from subject to subject. The experimenter learned the rate at which each subject reached those temperatures and adjusted the duration of initial rinsing accordingly.) At the end of this initial period of rinsing the subject placed the dorsal aspect of the tip of his/her tongue against the vibrator with just enough force to make full contact with the stimulator and surround. Tongue placement, which could be observed by the experimenter through the Plexiglas surround, was considered correct if the contactor was within about $1 \mathrm{~cm}$ of the end of the tongue. (It should be noted that more precise placement of the tongue is difficult to achieve because the softness of the lingual tissue causes the tip of the tongue to become indeterminate as it compresses against the surround.) The experimenter then monitored the temperature of the tongue via the digital thermometer, instructing the subject to resume rinsing if the temperature was too high or too low. To proceed with the trial, tongue temperature had to be either slightly below the target temperature (for cool and cold trials) or slightly above it (for warm trials). When this criterion had been met, the experimenter threw a switch to turn control of the trial sequence over to the computer. At the moment the temperature of the tongue moved to within $0.2^{\circ}-0.3^{\circ}$ of the target temperature, the computer initiated the trial.

Detection thresholds were measured using a two-alternative forced-choice staircase procedure (the up-down transformed method of Wetherill \& Levitt, 1965) with feedback. A trial began with presentation of broadband noise to the subject (through headphones) $0.5 \mathrm{sec}$ before the onset of the first of two 1-sec observation intervals. The observation intervals were separated by $0.5 \mathrm{sec}$ and their durations were signaled by LEDs. A 1-sec burst of vibration (gated at zero-crossing) occurred in one of the two intervals. The noise was turned off $0.5 \mathrm{sec}$ after the second interval, at which time the subjects responded by pressing one of two keys associated with the observation intervals. Immediately after the subject had responded, the LED of the "correct" interval flashed on, and the computer determined whether vibration amplitude should be increased, decreased, or stay the same for the next trial. The decision rule was the following: One incorrect-increase amplitude; one correct following an incorrect-no change; two correct in a row-decrease amplitude. The decision was displayed on a computer monitor and the experimenter made the required change (a 2-dB step) using the manual attenuators. While the experimenter adjusted stimulus intensity for the next trial, the subject rinsed again to maintain tongue temperature near the desired level. The procedure of tongue placement and temperature monitoring was repeated, and additional rinses were called for if the tongue had cooled or warmed too much since the last trial. After seven reversals of direction in the staircase procedure, the computer calculated the mean (in decibels) of the final six reversals, which was later converted into vibration amplitude in microns peak-to-peak (pk-pk).

An experimental session included three thresholds, one each at tongue temperatures of $36^{\circ}, 28^{\circ}$, and $20^{\circ} \mathrm{C}$. (These temperatures were chosen to span the range from normal oral temperature to the coldest temperature that could be maintained for several minutes without causing the teeth to ache.) The thresholds were run in an order of ascending or descending temperature, with 3 subjects tested in the descending sequence and 2 in the ascending sequence. Every subject contributed five thresholds at each temperature for two different frequencies of vibration: $30 \mathrm{~Hz}$ and $250 \mathrm{~Hz}$. The thresholds at each frequency were run in consecutive sessions, with 3 of the subjects tested first at the higher frequency and 2 tested first at the lower frequency.

\section{Results and Discussion}

Figure 1 shows the group means of the threshold amplitudes for the 30 - and $250-\mathrm{Hz}$ stimuli plotted as a function of tongue temperature. It is clear from these data that whereas the sensitivity of the anterior dorsal surface to $30-\mathrm{Hz}$ vibration was unaffected by cooling, the sensitivity to $250-\mathrm{Hz}$ vibration was greatly reduced at $20^{\circ} \mathrm{C}$. Every subject exhibited this pattern, and a twofactor ANOVA confirmed the existence of a main effect of temperature $[F(2,8)=51.54, p<.001]$ and a significant interaction between temperature and frequency $[F(2,8)=52.2, p<.001]$. Detection of the $250-\mathrm{Hz}$ vibration at a tongue temperature of $20^{\circ} \mathrm{C}$ required an

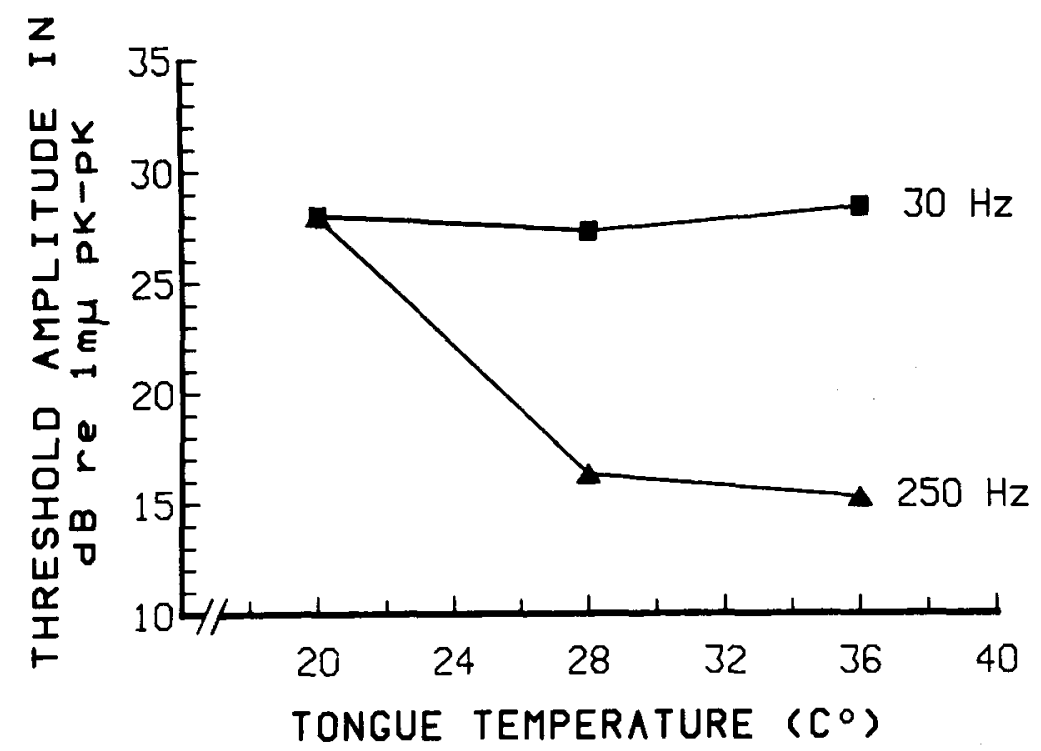

Figure 1. Vibrotactile detection thresholds measured on the anterior dorsal surface of the tongue at three temperatures. The parameter is the frequency of vibration. 
amplitude $12.1 \mathrm{~dB}$ higher than was required at a tongue temperature of $36^{\circ} \mathrm{C}(25.1 \mu \mathrm{m}$ vs. $6.2 \mu \mathrm{m})$. Similarly, the threshold at $20^{\circ} \mathrm{C}$ was $11.0 \mathrm{~dB}$ higher than it was at $28^{\circ} \mathrm{C}(25.1 \mu \mathrm{m}$ vs. $7.1 \mu \mathrm{m}) .{ }^{1}$ The difference between thresholds at $28^{\circ}$ and $36^{\circ}$ was not significant (Scheffé test, $p<.10$ ).

In addition to showing a strong cooling effect limited to the higher frequency, the threshold data represent another example of differential frequency sensitivity on the dorsal surface of the tongue when a rigid surround is used. At $36^{\circ} \mathrm{C}$, which approximates the normal temperature of the tip of the tongue, thresholds for the two frequencies differed by $13.0 \mathrm{~dB}$. These results, together with the results from other laboratories described in the introduction, imply that PCs can be excited by highfrequency vibration presented to the dorsal aspect of the anterior portion of the tongue, even when a surround is used.

The magnitude of the effect of cooling on the threshold for a $250-\mathrm{Hz}$ vibration was much greater than had been obtained on the tongue by Fucci et al. (1976). The strength of the effect in the present study is probably due to the use of a more stringent method of temperature control. As was noted above, Fucci et al. cooled the tongue with a single series of cold-water rinses before placing the tongue in a plastic clamp and measuring several detection thresholds in succession. It became apparent in the present experiment that the temperature of the tongue can rise several degrees per minute if it is not subjected to repeated cooling rinses (see also Pangborn, Chrisp, \& Bertolero, 1970).

It is notable that in the present study the effect of cooling was so strong that when skin temperature was lowered to $20^{\circ}$ the threshold for the $250-\mathrm{Hz}$ stimulus rose to the same amplitude as the threshold for the $30-\mathrm{Hz}$ stimulus. Because there was no measurable effect of cooling on the threshold for the $30-\mathrm{Hz}$ stimulus, the data imply that at $20^{\circ}$ the thresholds for both frequencies may have been mediated by the same afferent population(s). That is, the sensitivity of PCs may have been so reduced by cooling that they were no longer more sensitive to a $250-\mathrm{Hz}$ vibration than were other mechanoreceptors in the tongue, which normally mediate detection of lower frequencies of vibration.

\section{EXPERIMENT 2}

The purpose of Experiment 2 was to investigate the effect of cooling on the perceived intensity of vibration for the same two frequencies tested in Experiment 1. Of primary interest was whether the reduced sensitivity to the $250-\mathrm{Hz}$ stimulus caused by cooling would extend to suprathreshold amplitudes of vibration.

\section{Method}

Subjects. Twelve subjects, 9 females and 3 males (average age $=24.5$ years), were paid to participate. Five had served in Experiment 1 but did not know its outcome; none had had experience in judging suprathreshold vibrotactile stimuli.
Apparatus and Procedure. The apparatus used in Experiment 1 was used in the present experiment. The anterior dorsal surface of the tongue (within $1 \mathrm{~cm}$ of the tip) was again the site of testing, and the method of temperature control was unchanged from the first experiment.

The 7 subjects who had not participated in Experiment 1 were tested first in a brief session in which the thresholds for detecting 250- and $30-\mathrm{Hz}$ stimuli were determined with the temperature of the tongue held at $36^{\circ} \mathrm{C}$. These thresholds were needed to calculate vibration amplitudes corresponding to sensation levels (SLs) of $5,10,15,20,25$, and $30 \mathrm{~dB}$. Thresholds (measured using the two-alternative forced-choice staircase procedure used in Experiment 1) were determined once for each new subject at each frequency, and the thresholds obtained in Experiment 1 were used to set SLs for the remaining 5 subjects.

The procedure during a session was as follows: After rinsing the mouth with cold, cool, or warm water until the tongue was near the desired temperature (as determined by repeated temperature measurements), the subject placed the tongue against the contactor and surround. The experimenter turned control of the trial over to the computer, which triggered a trial sequence when the temperature of the tongue was within $0.2^{\circ}-0.3^{\circ} \mathrm{C}$ of the target temperature. The computer initiated a trial by turning on white noise $0.5 \mathrm{sec}$ before triggering the vibratory stimulus. An LED came on when the vibration did and stayed on throughout the 1.0-sec duration of the stimulus. (The LED served as a visual cue to help the subject attend to the vibration on trials in which the tongue was sufficiently desensitized by cooling to make detection of the weaker vibrations difficult.) The noise was turned off $0.5 \mathrm{sec}$ after termination of the stimulus, at which time the subject removed the tongue from the contactor and responded verbally with a magnitude estimate. (No modulus was given, and subjects practiced the method of magnitude estimation prior to beginning the task by assigning numbers to distances the experimenter produced between her hands.) Immediately after making a response, the subject rinsed three times with cold, cool, or warm water to maintain the tongue near the target temperature. An intertrial interval of $30 \mathrm{sec}$ was imposed to reduce the likelihood of adaption to the vibrotactile stimuli.

A session contained 54 trials: three repetitions of six vibration amplitudes (5-, 10-, 15-, 20-, 25-, and 30-dB SL) at each of three tongue temperatures $\left(20^{\circ}, 28^{\circ}\right.$, and $\left.36^{\circ} \mathrm{C}\right)$. The trials were blocked by temperature, with half of the subjects starting with a warm mouth $\left(36^{\circ}\right)$ and proceeding to the cooler temperatures and half starting with a cold mouth $\left(20^{\circ}\right)$ and proceeding to the warmer temperatures. Subjects served in two sessions, one for $30-\mathrm{Hz}$ stimuli and one for $250-\mathrm{Hz}$ stimuli.

\section{Results and Discussion}

The data for the 30- and $250-\mathrm{Hz}$ stimuli are displayed in Figures 2 and 3. Each data point is the arithmetic mean of normalized magnitude estimates. The data were normalized by dividing each subject's mean judgment at $36^{\circ}$ (calculated across vibration amplitudes) by the grand mean of all subjects at $36^{\circ}$ and then multiplying each subject's raw data by the resulting quotient.

It can be seen in Figure 2 that as was true at detection levels, cooling the tongue did not reduce the perceived intensity of the $30-\mathrm{Hz}$ vibration. [A two-factor ANOVA revealed no significant effect of temperature; $F(2,22)=$ $1.09, p=0.36$.] In contrast, Figure 3 displays a clear relationship between temperature and perceived magnitude for the $250-\mathrm{Hz}$ stimulus $[F(2,22)=111.0$, $p<.001$ ] . Cooling to $20^{\circ}$ produced such a decrement in sensitivity at $250 \mathrm{~Hz}$ that the two lowest amplitudes 


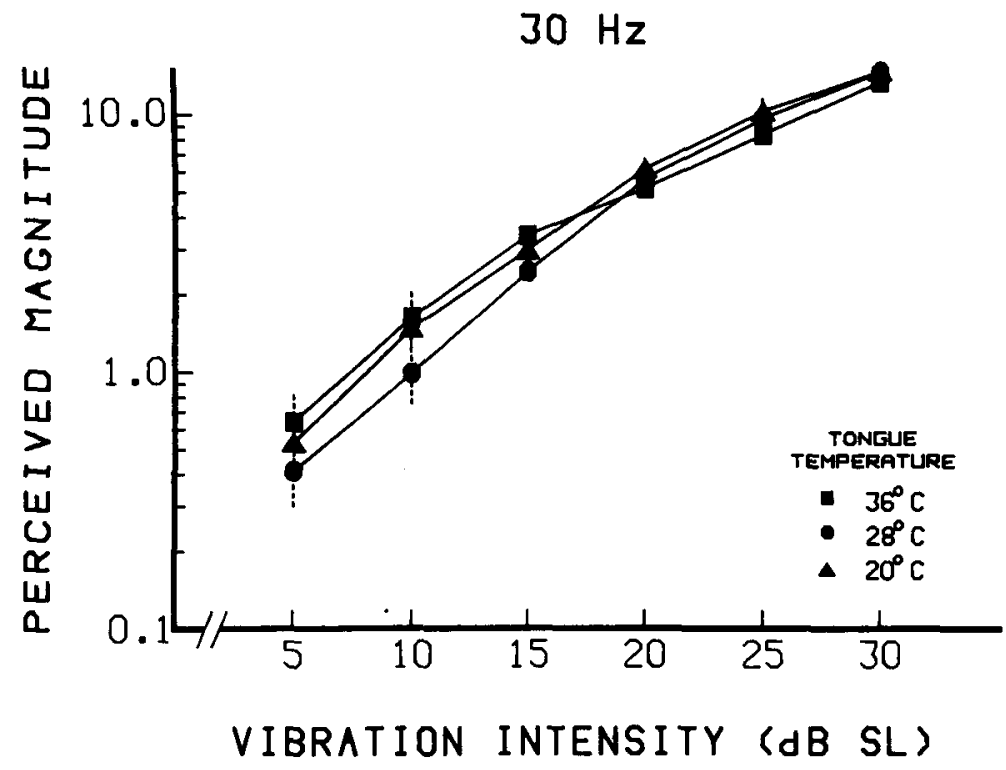

Figure 2. The perceived intensity of $30-\mathrm{Hz}$ vibration on the anterior dorsal surface of the tongue as a function of vibration intensity ( $\mathrm{dB}$ SL $=\mathrm{dB}$ above threshold amplitude). The parameter is tongue temperature. Vertical dashed lines indicated the standard errors of the means.

(5- and 10-dB SL re threshold at $36^{\circ}$ ) were perceived on fewer than half of the trials. The same was true for the lowest amplitude at $28^{\circ}$. Because these stimuli were perceived less than $50 \%$ of the time, they were considered below threshold and were dropped from the statistical analyses and from calculations of the best-fitting psychophysical functions. The inability to perceive $250-\mathrm{Hz}$ vibrations at SLs of $10 \mathrm{~dB}$ or less when the tongue was cooled to $20^{\circ}$ was consistent with the results of Experiment 1 . The threshold at $20^{\circ}$ in that experiment was shifted $12.1 \mathrm{~dB}$ higher than it was at $36^{\circ}$. In contrast, the results of Experiment 1 did not predict the failure to perceive the 5-dB-SL stimulus when the tongue was cooled to $28^{\circ}$. Cooling to $28^{\circ}$ produced a modest $1.2-\mathrm{dB}$ rise in threshold, which was not statistically significant. However, the threshold estimated from a two-alternative forced-choice detection task is likely to be lower than the "effective" threshold derived from a magnitude estimation task in which the subject is freer to set his/her own criterion for the presence of the signal.

Post hoc tests performed on the means at each vibration amplitude demonstrated that the source of the sig-

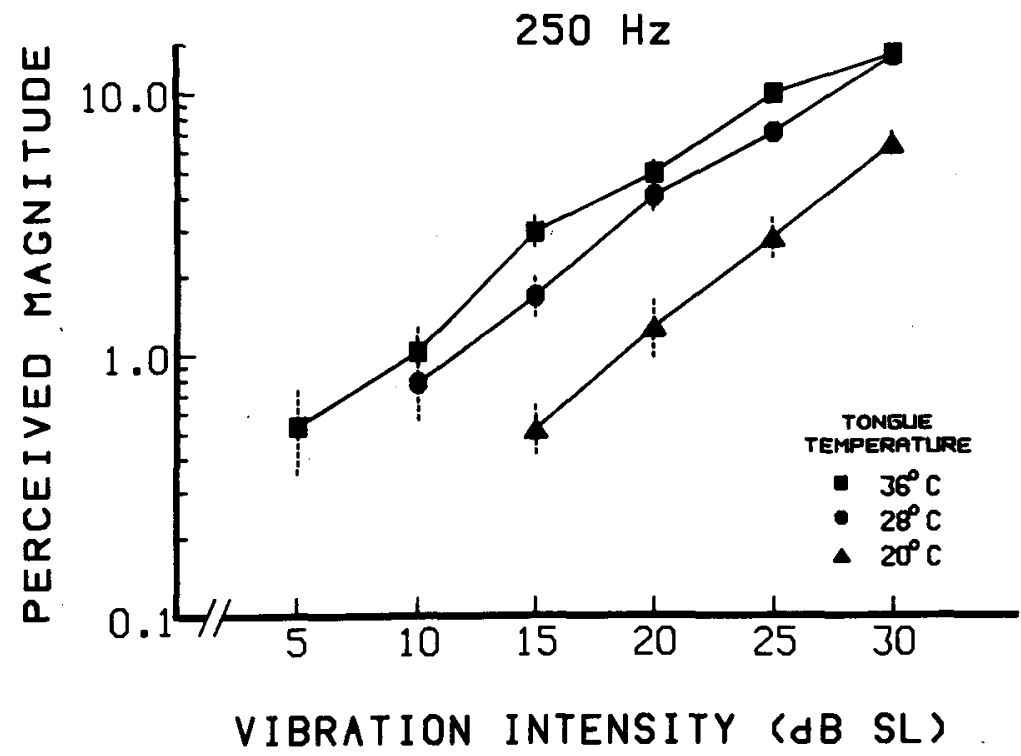

Figure 3. Same as Figure 2, but for 250-Hz vibration. 
nificance found with the ANOVA was the $20^{\circ} \mathrm{C}$ condition. Only at the $25-\mathrm{dB}$ SL was there a significant difference between perceived intensities at $36^{\circ}$ and $28^{\circ}$ (Tukey test, $p<.05$ ). In contrast, perceived intensity was significantly lower at every vibration amplitude when the tongue was cooled to $20^{\circ}$. A comparison of the data from the $20^{\circ}$ and $28^{\circ}$ conditions also revealed significant differences at the three highest amplitudes.

A comparison of the sensation magnitudes generated at $36^{\circ}$ and at $20^{\circ}$ indicates that cooling reduced the perceived intensity of vibration by more than $80 \%(0.52 \mathrm{vs}$. 2.98) at the lowest suprathreshold amplitude (15-dB SL, which translates into an average amplitude across subjects of $35 \mu \mathrm{m}$ pk-pk). The cooling effect grew smaller at higher amplitudes, but nevertheless caused nearly a $60 \%$ reduction (14.0 vs. 6.4) at the highest intensity tested (30$\mathrm{dB}$ SL, or an average amplitude of $197 \mu \mathrm{m}$ pk-pk). The inverse relationship between the size of the cooling effect and the amplitude of vibration is reflected by a significant temperature $\times$ amplitude interaction $[F(10,110)$ $=23.35, p<.001]$ and a difference in the exponents of the best-fitting power functions at $36^{\circ}$ and $20^{\circ}$. Calculated using the data for the four highest SLs, the exponents were $0.93(r=.992)$ at $36^{\circ}, 1.19(r=.995)$ at $28^{\circ}$, and $1.44(r=.999)$ at $20^{\circ}$. The steepness of the function at $20^{\circ}$ implies that at higher amplitudes the effect of temperature eventually becomes negligible. Projections of the $20^{\circ}$ and $36^{\circ}$ functions indicate that temperature should not affect perceived magnitude above about $40 \mathrm{~dB}$ SL.

The slope of the $250-\mathrm{Hz}$ function at $36^{\circ}$ is similar to the value reported for the upper portion of the psychophysical (power) function for the perceived intensity of a $250-\mathrm{Hz}$ vibration on the hand (e.g., Verrillo, Fraioli, $\&$ Smith, 1969), but higher than the values obtained on the dorsum of the tongue by Fucci and Petrosino (1982, 1983a, 1983b). (Fucci \& Petrosino, 1982, 1983a, reported that the slope of the upper limb of the function for a 250 $\mathrm{Hz}$ vibration was approximately 1.6; however, calculations based upon values obtained from their published graphs indicated that the slope was actually the reciprocal of that number, or approximately 0.65 .) Why the functions obtained in the present study are steeper than those obtained by Fucci and Petrosino is open to conjecture; there were many procedural differences between the studies. The two most notable differences were that Fucci and Petrosino used a magnitude production task rather than a magnitude estimation task, and a train of vibrotactile bursts (500-msec vibrations presented with a $50 \%$ duty cycle) rather than a single burst of vibration on each trial. Magnitude production, however, usually results in steeper psychophysical functions than does magnitude estimation, not shallower ones (Fucci, Harris, \& Petrosino, 1985; Verrillo et al., 1969). The use of a train of vibratory bursts (which apparently lasted throughout a series of six magnitude production trials) may have been more significant with regard to the slope of the function. Adjusting the intensity of a constantly pulsing stimulus would seem to be a very different task than assessing the magnitude of in- dividual bursts of vibration that are separated in time by tens of seconds.

\section{GENERAL DISCUSSION}

The data presented here demonstrate that cooling the tongue to $20^{\circ}$ causes a reduction in sensitivity to highfrequency $(250-\mathrm{Hz})$ vibration, but not to low-frequency $(30-\mathrm{Hz})$ vibration. This pattern of results is the same as has been found on the skin of the hand and the arm for threshold-level stimuli (Bolanowski \& Verrillo, 1982; Green, 1977; Verrillo \& Bolanowski, 1986), although there is evidence that more extreme cooling (to $15^{\circ} \mathrm{C}$ and below) may affect the sensitivity to low-frequency vibration as well (Bolanowski \& Verrillo, 1982).

The present results also constitute the first direct evidence obtained on any body locus that the effect of cooling remains significant at suprathreshold amplitudes of vibration. (A recent study, however, indicates that suprathreshold electrocutaneous sensations are also attenuated by cooling; Larkin \& Reilly, 1986.) Indirect evidence of a suprathreshold cooling effect for vibration was obtained in an earlier study (Green, Lederman, \& Stevens, 1979 ) in which it was found that suprathreshold sensations of roughness were attenuated when the fingertip was cooled. The latter observation, together with the present data, suggests that the perceived texture of ingested food is likely to be affected when the temperature of the tongue falls below normal. It is notable in this regard that the data of Green et al. (1979) indicate that perceived roughness begins to be affected at temperatures several degrees warmer than $20^{\circ} \mathrm{C}$, which may mean that oral texture perception is more vulnerable to cooling than is the perception of simple vibration.

A factor not controlled in the present experiments but which surely influences the strength of the cooling effect is the duration of exposure to the cooling stimulus. Vibrotactile perception may be undisturbed if exposure to cold ingesta is brief, simply because it takes time to cool the skin sufficiently to affect receptors that lie beneath the epidermis. Given that a robust effect of cooling is limited to high frequencies of vibration, exposure to the cold stimulus must presumably be of sufficient duration and extent to cool a population of mechanoreceptors (PCs) that may be restricted to the tissue deep within the ventral surface of the tongue (Spassova, 1965). It is even possible that transient exposure to cold ingesta enhances, rather than blunts, the perception of texture: Evidence from other experiments indicates that brief contact with a cold mechanical stimulus produces a tactile sensation that is both more intense and more spatially distinct than is the sensation produced by a thermally neutral stimulus (Stevens, 1979, 1982; Stevens \& Green, 1978). Future experiments will be needed to determine whether initial exposure to cold stimulation enhances lingual mechanoreception before longer exposures obtund it.

The present data also indicate that PCs may play a major role in the perception of vibration on the anterior dorsal surface of the tongue, even when a surround is present. 
This finding appears to conflict with the data of Verrillo $(1966,1968)$ but is consistent with some of the data of Fucci and his colleagues (e.g., Fucci \& Petrosino, 1982; Hall, Fucci, \& Arnst, 1972; Telage et al., 1972). One interesting feature of Verrillo's (1968) lingual data, however, is that the presence of a surround did not significantly decrease the sensitivity to high-frequency vibration. Instead, the surround increased the sensitivity to lowfrequency vibration, so much so that thresholds at all frequencies fell uniformly to the level of the threshold for the "best" frequency (about $250 \mathrm{~Hz}$ ) when no surround was present. In other words, because the threshold at $250 \mathrm{~Hz}$ was virtually the same whether or not a surround was used, it is unlikely the surround impeded the transmission of vibration to deep-lying PCs. Verrillo's results may be explained instead by the progressive increase in sensitivity to low-frequency vibration that Verrillo and his colleagues have since observed at other body loci when an "edge" is moved closer and closer to the mechanical driving point (Gescheider, Capraro, Frisina, Hamer, \& Verrillo, 1978; Verrillo, 1979; Verrillo \& Gescheider, 1979). By this interpretation, the surround provides an edge or spatial gradient that enhances perception of low frequencies of vibration. Why this may not always happen when a surround is used (as in the present study) is unclear. However, it cannot be ascertained whether or not in Experiment 1 the surround had at least a small effect on low-frequency thresholds, because no thresholds were measured without a surround. What does seem clear is that the relative contribution made by the $\mathrm{PC}$ and other mechanoreceptor systems to lingual vibrotactile sensitivity is not solely determined by the presence or absence of a surround. A number of other factors may be equally important, including, for example, the force with which the tongue contacts the vibrator and/or the surround, and the rostro-caudal location of stimulation. Experiments are planned that will investigate both of these variables.

We may conclude in the meantime that the vibrotactile sensitivity of the tongue is affected by cooling in much the same way as is vibrotactile sensitivity on hairy and glabrous skin, although a "significant" loss of sensitivity may occur only when exposure to cold stimulation is extensive and of long duration (i.e., several minutes). In addition, regardless of the reported absence of PCs in the dorsal surface of the tongue, the use of a rigid surround does not reliably eliminate the effect of frequency on vibrotactile sensitivity.

\section{REFERENCES}

Bartoshuk, L. M., Rennert, K., Rodin, J., \& Stevens, J. C. (1982). Effects of temperature on the perceived sweetness of sucrose. Physiology \& Behavior, 28, 905-910.

Bolanowski, S. J., JR., \& VerRillo, R. T. (1982). Temperature and criterion effects in a somatosensory subsystem: A neurophysiological and psychophysical study. Journal of Neurophysiology, 48, 836-855.

Calviño, A. M. (1986). Perception of sweetness: The effects of concentration and temperature. Physiology \& Behavior, 36, 1021-1028.

FuCCI, D. J. (1972). Oral vibrotactile sensation: An evaluation of nor- mal and defective speakers. Journal of Speech \& Hearing Research, 15, 179-184.

Fucci, D. J., Blackmon, R. C., Lindsey, S., \& MCCaffrey, P. (1974). The effect of age on vibrotactile thresholds obtained from the tongue. Ohio Journal of Speech \& Hearing, 10, 18-24.

Fucci, D. J., Crary, M. A., \& Telage, K. M. (1977). Lingual clamping procedures for measuring oral vibrotactile thresholds: II. Effects of using a lower clamping disk. Bulletin of the Psychonomic Society, $10,457-459$.

Fucci, D., Crary, M., Wilson, H., \& Curtis, A. P. (1976). Vibrotactile and temperature sensory interaction in the human tongue. Perceptual \& Motor Skills, 43, 263-266.

Fucci, D. J., Curtis, A. P., Harnack, M. M. (1974). Oral vibrotactile stimulation: A method for monitoring change in lingual sensitivity as a function of time. Bulletin of the Psychonomic Society, 4, 573-574.

FucCI, D. J., Hall, D. E., \& Weiner, F. F. (1971). Normative study of oral and non-oral structures using vibrotactile stimuli. Perceptual \& Motor Skills, 33, 1099-1105.

FucCi, D., Harris, D., \& Petrosino, L. (1985). Threshold and suprathreshold correlations for the oral tactile sensory mechanism. Journal of Speech \& Hearing Research, 28, 331-335.

FucCI, D., \& PeTrosino, L. (1982). Lingual vibrotactile sensation magnitudes: Stimulus frequency effect. Bulletin of the Psychonomic Society, 20, 224-226.

FucCI, D., \& Petrosino, L. (1983a). Lingual vibrotactile sensation magnitudes: Comparison of suprathreshold responses for the tongue and hand. Journal of the Acoustical Society of America, 74, 351-353.

FuCcI, D., \& PeTrosino, L. (1983b). Lingual vibrotactile sensation magnitudes: Comparison of suprathreshold responses in men and women. Perception \& Psychophysics, 33, 93-95.

Gescheider, G. A., Capraro, A. J., Frisina, R. D., Hamer, R. D., \& Verrillo, R. T. (1978). The effects of a surround on vibrotactile thresholds. Sensory Processes, 2, 99-115.

GreEN, B. G. (1977). The effect of skin temperature on vibrotactile sensitivity. Perception \& Psychophysics, 21, 243-248.

GREEN, B. G. (1986). Sensory interactions between capsaicin and temperature in the oral cavity. Chemical Senses, 11, 371-382.

Green, B. G., Frankmann, S. P. (in press). The effect of cooling the tongue on the perceived intensity of taste. Chemical Senses.

Green, B. G., Lederman, S. J., \& Stevens, J. C. (1979). The effect of skin temperature on the perception of roughness. Sensory Processes, 3, 327-333.

HALL, D. E., Fucci, D. J., \& ARNST, D. J. (1972). Vibrotactile stimulation: An investigation of psychophysical methods for establishing threshold. Perceptual \& Motor Skills, 34, 891-898.

Inman, D. P., \& Peruzzi, P. (1961). The effect of temperature on the responses of Pacinian corpuscles. Journal of Physiology, 155, 280-301.

IsHIKo, N., \& LOEWENSTEIN, W. R. (1961). Effects of temperature on the generator and action potentials of a sense organ. Journal of General Physiology, 45, 105-124.

KoradeCKA, D. (1974). Changes in the threshold of vibration sensibility depending on skin temperature. Acta Physiologica Poland, 25, 207-214.

LARKIN, W. D., \& ReILLY, J. P. (1986). Electrocutaneous sensitivity: Effects of skin temperature. Somatosensory Research, 3, 261-271.

McBurney, D. H., Collings, V. B., \& Glanz, L. M. (1973). Temperature dependence on human taste responses. Physiology \& Behavior, 11, 89-94.

Pangborn, R. M., Chrisp, R. P., \& Bertolero, L. L. (1970). Gustatory, salivary and oral thermal responses to solutions of sodium chloride at four temperatures. Perception \& Psychophysics, 8, 69-75.

SHERRICK, C. E., JR. (1953). Variables affecting sensitivity of the human skin to mechanical vibration. Journal of Experimental Psychology, 45, 273-282.

SIZER, F., \& HARRIs, N. (1985). The influence of common food additives and temperature on threshold perception of capsaicin. Chemical Senses, 10, 279-286.

SPASSOVA, I. (1965). On the structure of encapsulated nerve endings in tongue of the cat and its functional significance. Zeitschrift für Mikroskopisch-Anatomische Forschung, 72, 366-382. 
Stevens, J. C. (1979). Thermal intensification of touch sensation: Further extensions of the Weber phenomenon. Sensory Processes, 3, 240-248.

Stevens, J. C. (1982). Temperature can sharpen tactile acuity. Perception \& Psychophysics, 31, 577-580.

Stevens, J. C., \& Green, B. G. (1978). Temperature-touch interaction: Weber's phenomenon revisited. Sensory Processes, 2, 206-219.

Telage, K. M., Fucci, D. J., \& ARNST, D. J. (1972). Normative study of oral vibrotactile sensitivity. Perceptual \& Motor Skills, 35, 671-676.

Telage, K. M., Fucci, D., \& Blackmon, R. (1976). Temporal summation effects on lingual vibrotactile thresholds. Perceptual \& Motor Skills, 42, 859-864.

Telage, K. M., \& Petrosino, L. A. (1978). Testing procedures for measuring oral vibrotactile thresholds: III. Effects obtained using a nonclamping procedure. Bulletin of the Psychonomic Society, 12, 291-293.

VerRILlo, R. T. (1966). Stimulus specificity in a cutaneous receptor. Perception \& Psychophysics, 1, 149-159.

VerRILLO, R. T. (1968), A duplex mechanism of mechanoreception. In D. R. Kenshalo (Ed.), The skin senses (pp. 139-159). Springfield, IL: Thomas.

VerRILLO, R. T. (1979). The effect of surface gradients on vibrotactile thresholds. Sensory Processes, 3, 27-36.

VerRILLo, R. T., \& BolaNowskI, S. J. (1986). The effects of skin temperature on the psychophysical responses to vibration on glabrous and hairy skin. Journal of the Acoustical Society of America, 80, 528-532.

Verrillo, R. T., Fraioli, A. J., \& SMITH, R. L. (1969). Sensation magnitude of vibrotactile stimuli. Perception \& Psychophysics, 6 , 366-372.

Verrillo, R. T., \& GescheIDER, G. A. (1979). Psychophysical measurements of enhancement, suppression and surface gradient effects in vibrotaction. In D. R. Kenshalo (Ed.), Sensory functions of the skin of humans (pp. 153-181). New York: Plenum Press.

WeITZ, J. (1941). Vibrotactile sensitivity as a function of skin temperature. Journal of Experimental Psychology, 28, 21-36.

WETHERILL, G. B., \& LEVITT, H. (1965). Sequential estimation of points on a psychometric function. The British Journal of Mathematical \& Statistical Psychology, 18, 1-10.

\section{NOTE}

1. These threshold values lie toward the high end of a wide range of amplitudes (spanning about $\mathbf{4 0} \mathrm{dB}$ ) reported for the dorsal surface of the tongue. Among the thresholds reported by Fucci and his co-workers for vibrations of approximately $200-250 \mathrm{~Hz}$ are $0.075 \mu \mathrm{m}$ (Hall, Fucci, $\&$ Arnst, 1972), $0.4 \mu \mathrm{m}$ (Telage, Fucci, \& Arnst, 1972), $1.3 \mu \mathrm{m}$ (Fucci, Harris, \& Petrosino, 1985) and 2.1 to $2.8 \mu \mathrm{m}$ (Fucci, Crary, \& Telage, 1977). Although not specified, it is assumed these values represent peakto-peak (pk-pk) amplitudes. Verrillo (1968) reported a threshold of about $4.5 \mu \mathrm{m}$ peak $(9.0 \mu \mathrm{m}$ pk-pk) using a contactor 10 times larger than the one employed by Fucci and the others $\left(1.3 \mathrm{~cm}^{2}\right.$ vs. $\left.0.128 \mathrm{~cm}^{2}\right)$. All of the above measurements were made with a rigid surround, and some had a bottom (clamping) surface in addition to the surround. Sherrick (1953) obtained a threshold on the tip of the tongue of 3-4 $\mu \mathrm{m}$ pk-pk without a surround and using a contactor $0.137 \mathrm{~cm}^{2}$ in area.

(Manuscript received December 1, 1986; revision accepted for publication April 15, 1987.) 